\title{
Stretchable piezoelectric nanocomposite generator
}

\author{
Kwi-ll Park ${ }^{1 *+}$, Chang Kyu Jeong ${ }^{2,3+}$, Na Kyung Kim² and Keon Jae Lee ${ }^{2^{*}}$
}

\begin{abstract}
Piezoelectric energy conversion that generate electric energy from ambient mechanical and vibrational movements is promising energy harvesting technology because it can use more accessible energy resources than other renewable natural energy. In particular, flexible and stretchable piezoelectric energy harvesters which can harvest the tiny biomechanical motions inside human body into electricity properly facilitate not only the self-powered energy system for flexible and wearable electronics but also sensitive piezoelectric sensors for motion detectors and in vivo diagnosis kits. Since the piezoelectric ZnO nanowires (NWs)-based energy harvesters (nanogenerators) were proposed in 2006, many researchers have attempted the nanogenerator by using the various fabrication process such as nanowire growth, electrospinning, and transfer techniques with piezoelectric materials including polyvinylidene fluoride (PVDF) polymer and perovskite ceramics. In 2012, the composite-based nanogenerators were developed using simple, lowcost, and scalable methods to overcome the significant issues with previously-reported energy harvester, such as insufficient output performance and size limitation. This review paper provides a brief overview of flexible and stretchable piezoelectric nanocomposite generator for realizing the self-powered energy system with development history, power performance, and applications.
\end{abstract}

Keywords: Energy harvesting, Self-powered system, Piezoelectric, Stretchable nanogenerator, Flexible, Composite

\section{Background}

Attractive approaches based on energy harvesting technology that convert ambient energy resources such as thermal, solar and mechanical energies into electrical energy have been recently studied to realize the demonstration of self-powered energy system in portable devices without external power sources like batteries [1-4]. Among these sustainable energy resources, the mechanical energy is easily accessible compared to outdoor renewable energy in anytime and anywhere (even inside human body) of our daily [5-8]. To convert the mechanical energy resources (e.g., pressure, bending, stretching and vibrational motions) to electricity,

\footnotetext{
*Correspondence: kipark@gntech.ac.kr; keonlee@kaist.ac.kr

${ }^{\dagger}$ Kwi-II Park and Chang Kyu Jeong contributed equally to this work

${ }^{1}$ Department of Energy Engineering, Gyeongnam National University of Science and Technology (GNTECH), 33 Dongjin-ro, Jinju-si,

Gyeongsangnam-do 52725, Republic of Korea

2 Department of Materials Science and Engineering, Korea Advanced Institute of Science and Technology (KAIST), 291 Daehak-ro, Yuseong-gu, Daejeon 34141, Republic of Korea

Full list of author information is available at the end of the article
}

the piezoelectric energy conversion are proposed and investigated by many research groups $[3,9,10]$. In particular, the flexible energy harvester called a nanogenerator which can generate electrical energy from not only mechanical energy but also tiny biomechanical energy (e.g., heartbeat, muscle motions, and eye blinking) have attracted attention in response to the demands of infinite self-powered sources for operating flexible and wearable electronic systems [11].

The first nanogenerator is the piezoelectric $\mathrm{ZnO}$ nanowires (NW) based energy device proposed by Wang and co-workers, as shown in Fig. 1a [12-15]. A single $\mathrm{ZnO} \mathrm{NW}$ on a flexible polyimide (PI) substrate successfully convert from the human finger motions (Fig. 1a-i) and the running/scratching motions of a hamster to electricity $[12,13]$. They also have used the transferred lateral $\mathrm{ZnO} \mathrm{NW}$ arrays on a flexible substrate to enhance the output performance of nanogenerator [14], that have provided the technical advancement to operate commercial electronic devices using the nanogenerator technology (Fig. 1a-ii). Choi et al. [15] reported the transparent and 


\section{a $\mathrm{ZnO}$ Nanowire-based Nanogenerators}

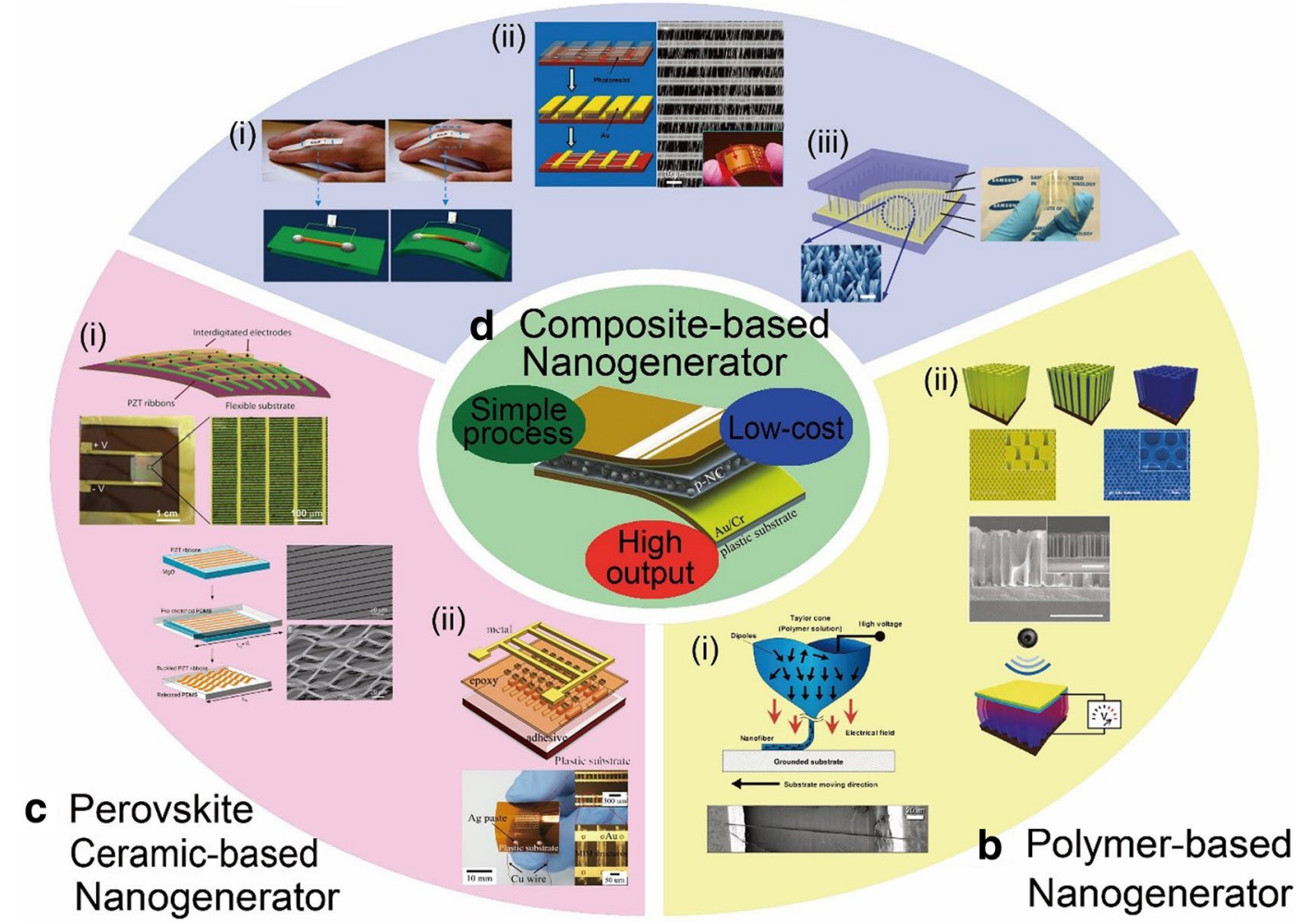

Fig. 1 Previously reported flexible energy harvesting devices. Nanogenerators based piezoelectric materials such as (a) ZnO NW (reproduced from ref. [12, 14, 15] with permission, American Chemical Society and Wiley-VCH). b PVDF polymer (reproduced from ref. [16, 18] with permission, American Chemical Society) c Perovskite ceramic thin film (reproduced from ref. [19, 21, 22] with permission, American Chemical Society and Royal Society of Chemistry)

flexible nanogenerator which involves the $\mathrm{ZnO}$ nanorod (NR) arrays and indium tin oxide (ITO) electrodes coated polyether sulfone (PES) flexible substrates (Fig. 1a-iii).

Because polymer materials have the naturally flexible properties and mechanical stabilities, the piezoelectric polymers as polyvinylidene fluoride (PVDF) have been used to fabricate polymeric flexible nanogenerators (Fig. 1b) [16-18]. Figure 1b-i presents the piezoelectric PVDF nanofibers placed on a working substrate using a unique direct-write technique. The PVDF based nanogenerator generates electrical outputs of about $5-30 \mathrm{mV}$ and $0.5-3 \mathrm{nA}$ when the substrate is deformed by stretching and releasing motions $[16,17]$. Cha et al. [18] proposed the polymeric nanogenerator made of porous PVDF by employing a novel template-assisted method (Fig. 1b-ii). This sonic wave driven energy harvester produces higher output performance compared to dense PVDF based nanogenerators due to the effective nanoporous structure.

In 2010, there have been new approaches to use perovskite-structured ceramic $\left[\mathrm{PbZr}_{\mathrm{x}} \mathrm{Ti}_{1-\mathrm{x}} \mathrm{O}_{3}\right.$ (PZT) and $\mathrm{BaTiO}_{3}$ ] thin films with inherently high piezoelectricity for higher energy conversion efficiency (Fig. 1c) [1922]. The PZT and $\mathrm{BaTiO}_{3}$ thin films on bulk substrate are transferred onto flexible PI substrates by adopting unique transferring techniques after high temperature crystallization process (Fig. 1c-i, ii). The perovskite thin film-based nanogenerators show higher power density compared with other flexible piezoelectric devices with the similar device structure. Qi et al. [21] presented the energy harvester of wavy piezoelectric PZT ribbons, that can scavenge stretching motions to generate.

Recently, the research on large-area, low-cost, mechanically-stable, and high-output nanocomposite generator has been invented by using simply casting piezoelectric nanocomposites onto flexible plastic substrates at low temperature (Fig. 1d) [23-27]. In particular, several research groups have developed lead-free and highperformance flexible energy harvesters using bio-ecocompatible piezoelectric ceramics such as $\mathrm{BaTiO}_{3}$ [23], $(\mathrm{K}, \mathrm{Na}) \mathrm{NbO}_{3}$ [24, 25], and Li-doped (K, Na) $\mathrm{NbO}_{3}$ [27]. Subsequently, a new concept of ultra-stretchable elasticcomposite generator, was also developed by employing the Ecoflex silicone rubber-based piezoelectric 
composites and long silver nanowire-based stretchable electrodes [28]. This review paper highlights a brief overview of flexible and stretchable piezoelectric nanocomposite generator for realizing the self-powered energy system, summarizing development history, power performance, and energy applications. These technologies provide novel solutions to overcome the challenging issues about nanogenerators, such as insufficient output signals and size limitation for the power sources of commercial electronics.

\section{Review}

\section{1 $\mathrm{BaTiO}_{3}$ nanoparticles-based flexible nanocomposite generator}

Park et al. firstly demonstrated the nanocompositebased nanogenerator (NCG) using piezoelectric $\mathrm{BaTiO}_{3}$ nanoparticles and universal graphitic carbons [such as carbon nanotube (CNT) and reduced graphene oxide] by employing simple, low-cost, and large-area spincasting/bar-coating method (Fig. 2a) [23]. A piezoelectric nanocomposite (p-NC) was produced by simply dispersing the piezoelectric $\mathrm{BaTiO}_{3}$ nanoparticles (NPs) and graphitic carbons within a polydimethylsiloxane (PDMS) elastomer. Note that the graphitic carbons in the NCG device play a role as dispersant avoiding precipitation of $\mathrm{BaTiO}_{3} \mathrm{NPs}$, stress agent reinforcing piezomaterials, and electrical nanobridge forming conduction paths in the polymer matrix. As shown in Fig. 2b and c, the $\mathrm{p}-\mathrm{NC}$ sandwiched between electrode-coated plastic substrates has the thickness of $300 \mu \mathrm{m}$ and the welldispersed nanomaterials. Figure $2 \mathrm{~d}$ presents a $3 \times 3 \mathrm{~cm}^{2}$ sized-NCG device which can be bent by human fingers owing to the naturally flexible properties of $\mathrm{p}-\mathrm{NC}$. Figure $2 \mathrm{e}$ and $\mathrm{f}$ show the power generation mechanism of NCG device and the calculated piezopotential inside $\mathrm{p}-\mathrm{NC}$ which is produced by mechanical deformation. During the regularly mechanical bending and unbending deformations, the electrons move up and down between the top and bottom electrodes; as a results, these repeat flows can generate positive and negative electric pulses (Fig. 2e). To confirm the power generation of the NCG device, the simple model consisting of six $\mathrm{BaTiO}_{3} \mathrm{NPs}$ in PDMS matrix was established and calculated by multiphysics COMSOL software. Since the entire p-NC layer is tensile-stressed by bending NCG device, the piezoelectric potential is generated across the electrodes due to the piezoelectric effect of NPs (Fig. 2f). This technology can offer a significant scientific advancement for flexible piezoelectric energy harvester since it removes the demerits of previous nanogenerator such as size limitation and cost issues.

\subsection{NCG device based on various piezoelectric particles}

To characterize the output performance of NCG device, a customized bending machine of deforming the flexible devices was utilized, as shown in Fig. 3a-i. The $100 \mathrm{~nm}$ sized $\mathrm{BaTiO}_{3}$ NPs (Fig. 3a-ii)-based NCG device made an open-circuit voltage of $\sim 3.2 \mathrm{~V}$ and a short-circuit current of 250-350 nA (Fig. 3a-iii, iv) during the repeatedly bending motions corresponding to displacement of $5 \mathrm{~mm}$ from original $4 \mathrm{~cm}$ long sample at strain rate of $0.2 \mathrm{~m} \mathrm{~s}^{-1}$ [23].

Since the first demonstration of $\mathrm{BaTiO}_{3}$ NPs-based NCG device, many research groups proposed other type of composite-based nanogenerators composed of various piezoelectric materials such as $\mathrm{ZnO}, \mathrm{PZT}$, and alkaline niobate particles using the NCG technique [26, 27, 29], due to the advantage of highly-efficient and large-area energy harvesters with simple and low-cost process. By using $\mathrm{ZnO}$ NPs of the higher piezoelectric performance compared to $\mathrm{ZnO}$ nanowire, the novel flexible nanogenerator composed of $\mathrm{ZnO}$ NPs and multiwall-carbon nanotubes (MW-CNTs) was demonstrated, as shown in Fig. 3b [29]. The energy harvester generated output voltage of $0.4 \mathrm{~V}$ and current pulse of $50 \mathrm{nA}$ during the repeat finger gestures with the frequency of $1 \mathrm{~Hz}$. The voltage and current signals reached up to the $7.5 \mathrm{~V}$ and $2.5 \mu \mathrm{A}$, respectively, under hammer knocking on the energy device. The inherently excellent piezoelectric PZT particles were also adopted to fabricate NCG device (Fig. 3c) [26]. The NCG device with PZT particles and multiwall (MW)-CNTs showed the distinguishable improvement in output performance of NCG device $\left(3 \times 3 \mathrm{~cm}^{2}\right)$ : the converted output voltage and current signals are $\sim 10 \mathrm{~V}$ and $1.3 \mu \mathrm{A}$, respectively. Although this nanogenerator provided the methodology for high-output flexible energy harvesting device, PZT-based device has critical environmental drawbacks due to $\mathrm{Pb}$-related problems. One of the most attractive lead-free piezoelectric materials is alkaline niobate which provides similar piezoelectric properties compared to PZT material. Jeong et al. [27] constructed the lead-free NCG device made of outstanding piezoelectric and bio-ecofriendly $(\mathrm{K}, \mathrm{Na}) \mathrm{NbO}_{3}-$ $\mathrm{LiNbO}_{3}$ (KNLN) nanoparticles and well-dispersible copper nanorods (NRs) fillers (Fig. 3d). The KNLN-based NCG device not only creates high output with $12 \mathrm{~V}$ and $1.2 \mu \mathrm{A}$ but also shows excellent stability and durability without any degeneration under the repeated bending cycles. By employing the bar-coating method, a largearea NCG device of $30 \times 30 \mathrm{~cm}^{2}$ was demonstrated, as shown in Fig. 3e [26]. A large-scale NCG device harvested high output signals, output voltage of $\sim 100 \mathrm{~V}$ and current of $\sim 10 \mu \mathrm{A}$. 

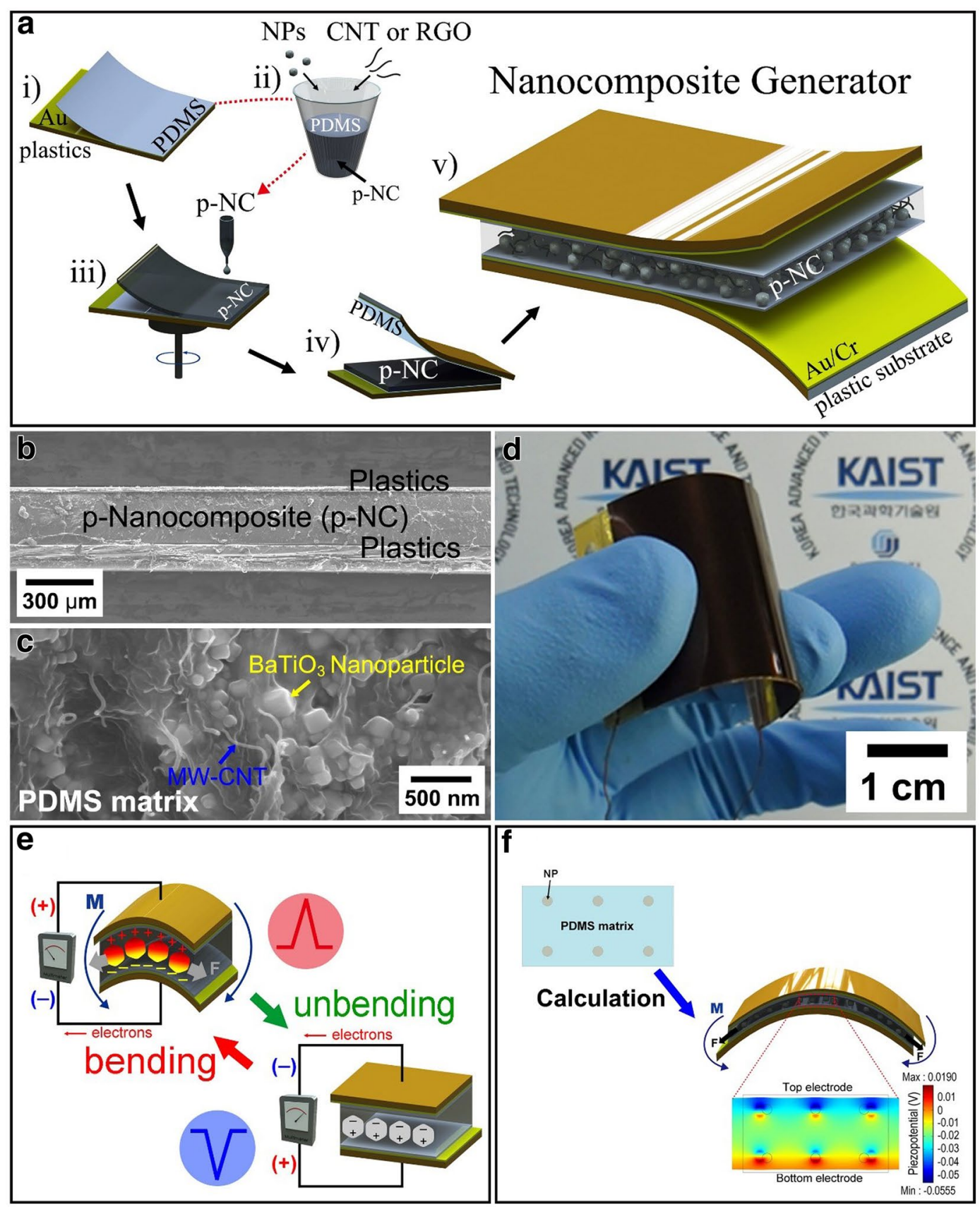

Fig. 2 Flexible nanocomposite-based generator made of $\mathrm{BaTiO}_{3} \mathrm{NPs}_{\text {and }}$ graphitic carbons. a Schematic illustration showing the fabrication process of an NCG device by simple, low-cost, and scalable spin-casting. b A cross-sectional SEM image of an NCG device. c The magnified photograph of piezoelectric nanocomposite. d The fabricated NCG device composed of p-NC and electrode-coated plastic substrates. e Schematics showing power generation mechanism of the NCG device. $\mathbf{f}$ Simulation model of an NCG device consisted of six $\mathrm{BaTiO}_{3} \mathrm{NPs}$ inside PDMS matrix and the calculated piezoelectric potential inside the p-NC method (reproduced from ref. [23] with permission, Wiley-VCH)

\subsection{Piezoelectric nanowire/tubes-based NCG device}

To demonstrate the high-output NCG devices based on piezoelectric NPs, the toxic dispersant such as MWCNTs and $\mathrm{Cu}$ NRs should be inevitably used to avoid the aggregation of piezoelectric NPs and enhance the output performance of electrical generation. Some research groups developed the lead-free and eco-friendly NCG devices made of non-toxic piezoelectric one-dimensional nanostructure (i.e., nanowire and nanotube) without harmful dispersing agents. Jung et al. reported the NCG 


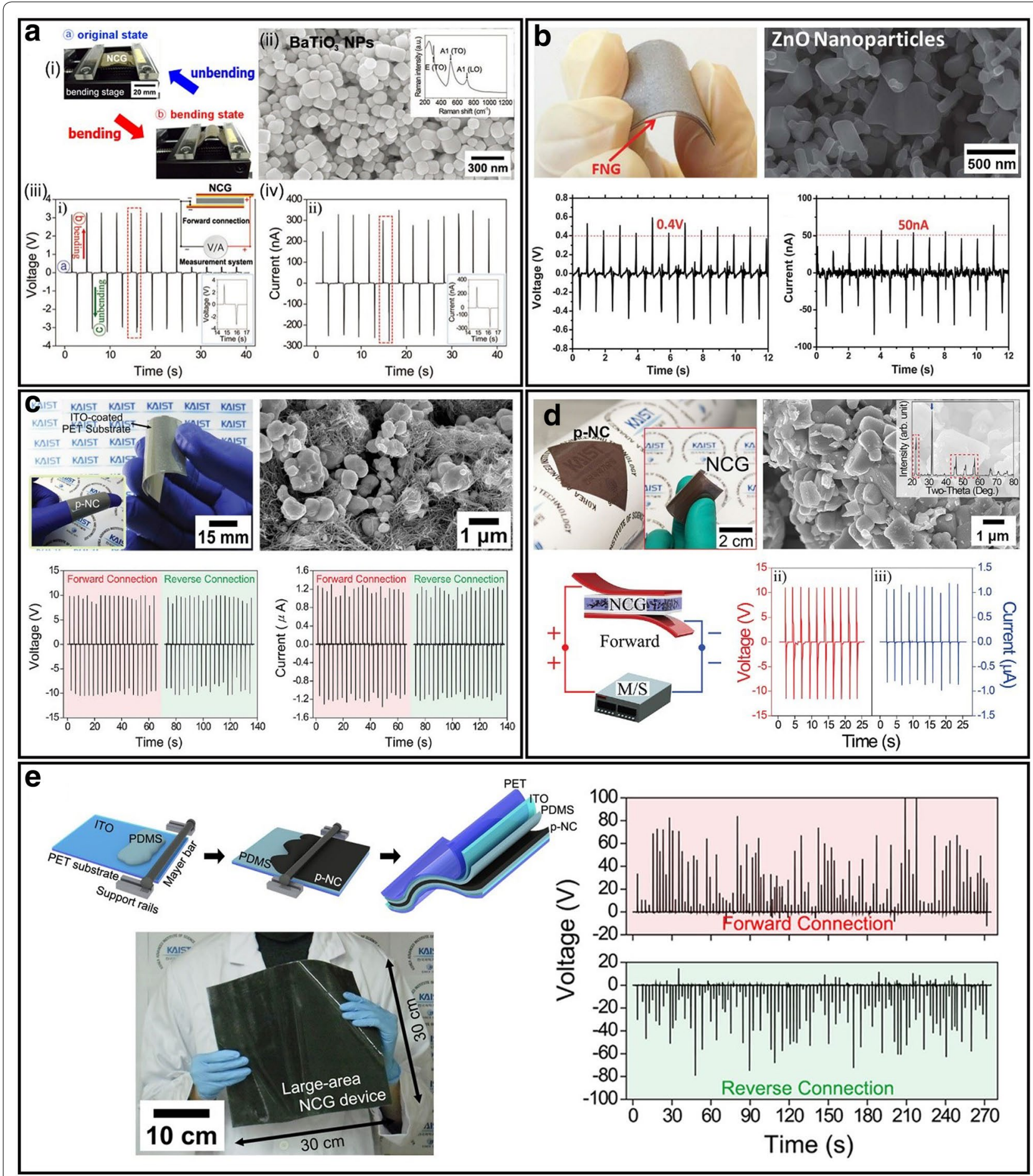

Fig. 3 The fabricated NCG devices based on various piezoelectric particles. The generated output voltage and current signals from a $\mathrm{BaTiO}_{3} \mathrm{NPS}_{\mathrm{S}}$ (reproduced from ref. [23] with permission, Wiley-VCH), b ZnO NPs (reproduced from ref. [29] with permission, Royal Society of Chemistry), c PZT particles (reproduced from ref. [26] with permission, Wiley-VCH), KNLN particles (d) (reproduced from ref. [27] with permission, Wiley-VCH) based NCG device under periodically bending motions. e The large-area NCG device fabricated by a bar-coating technique and the measured output performance (reproduced from ref. [26] with permission, Wiley-VCH) 
device using lead-free $\mathrm{NaNbO}_{3} \mathrm{NWs}$ [24] and $\mathrm{KNbO}_{3}$ NRs [25] as shown in Fig. 4a and b, respectively. $\mathrm{NaNbO}_{3}$ $\mathrm{NWs}$ and $\mathrm{KNbO}_{3} \mathrm{NRs}$ were synthesized via hydrothermally grown method at low temperature; as a result, $\mathrm{NaNbO}_{3} \mathrm{NWs}$ and $\mathrm{KNbO}_{3}$ NRs showed the length of $\sim 10 \mu \mathrm{m}$ with diameter of $\sim 200 \mathrm{~nm}$ and length of $\sim 1 \mu \mathrm{m}$, respectively. The lead-free alkaline-based piezoelectric materials-PDMS polymer composites were sandwiched by top and bottom electrodes-coated PI substrates and then were bent by periodically mechanical agitations. Consequently, the two NCG device converted similar output performance with open-circuit voltage of $3.2 \mathrm{~V}$ and $\sim 70 \mathrm{nA}$. Figure $4 \mathrm{c}$ and d show the bio-eco-compatible NCG devices achieved by using the lead-free piezoelectric $\mathrm{BaTiO}_{3}$ nanotube [30] or nanowires [31]. Lin et al. [30] synthesized the $\mathrm{BaTiO}_{3}$ nanotubes by hydrothermal method and formed the $\mathrm{p}-\mathrm{NC}$ by dispersing process. The flexible and transparent $\mathrm{BaTiO}_{3}$ nanotubes-based harvester converted the outputs of $5.5 \mathrm{~V}$ and $350 \mathrm{nA}$ under a stress of $1 \mathrm{MPa}$ (Fig. 4c). The lead-free piezoelectric $\mathrm{BaTiO}_{3} \mathrm{NWs}$ synthesized by hydrothermal method at low temperature shows the average length of $\sim 4 \mu \mathrm{m}$ with a high aspect ratio (Fig. 4d) [31]. The $\mathrm{BaTiO}_{3}$ NWs could be well-distributed in PDMS elastomer without dispersing agents. Under the periodically bending and unbending motions, the output voltage and current generated from NCG device were $\sim 7.0 \mathrm{~V}$ and $\sim 360 \mathrm{nA}$, respectively. These measured values are higher than those of previous NWs-based NCGs: the high energy conversion is caused by adopting the nanostructures with high aspect ratio which can be well distributed in PDMS matrix without any dispersant agent.
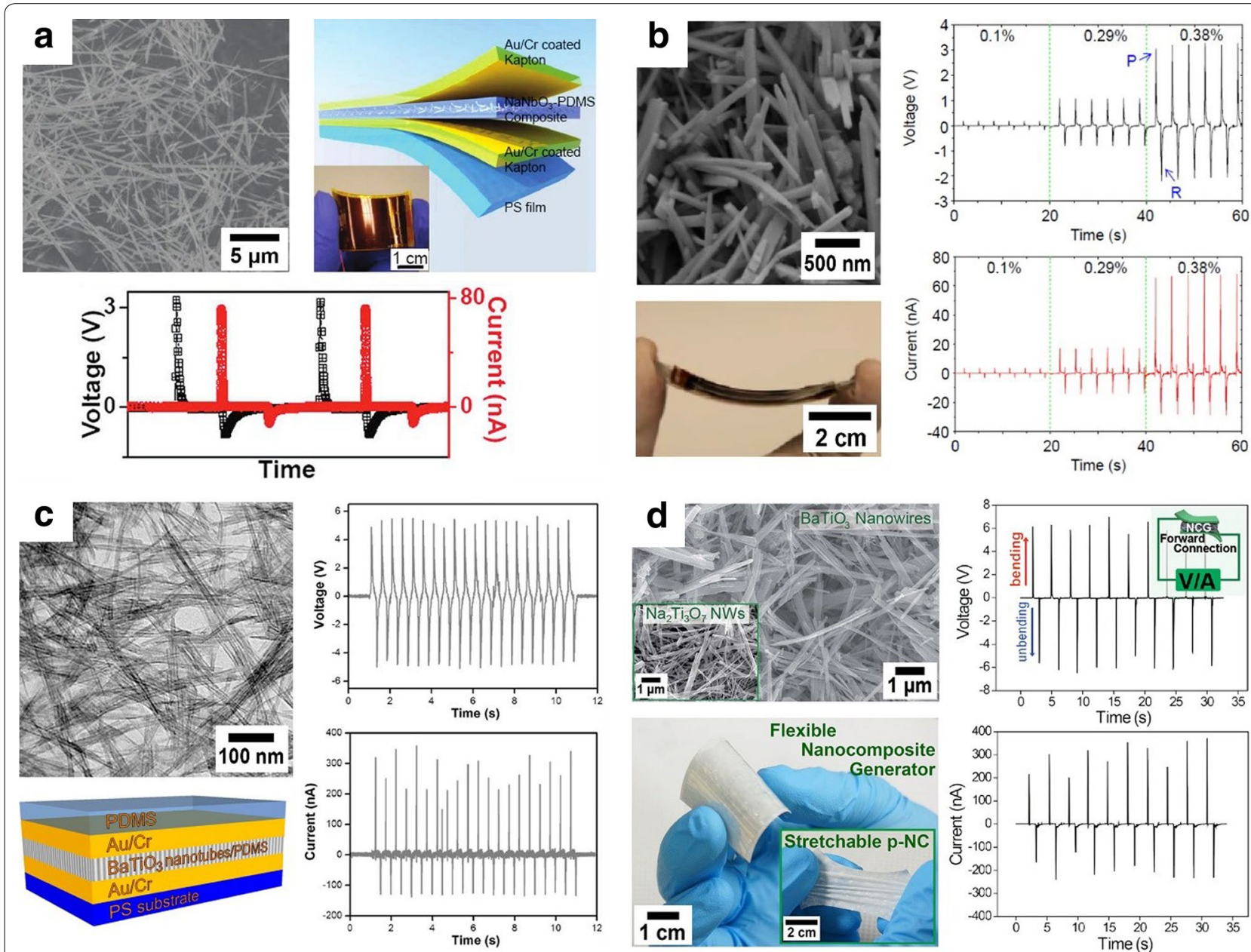

Fig. 4 The only piezoelectric nanowires/tubes-based NCG devices. The photographs and output performance of energy harvesters made of $\mathrm{NaNbO}_{3} \mathrm{NWs}$ (a) (reproduced from ref. [24] with permission, American Chemical Society), $\mathrm{KNbO}_{3} \mathrm{NRs}$ (b) (reproduced from ref. [25] with permission, IOP Publishing), $\mathrm{BaTiO}_{3} \mathrm{NTs}$ (c) (reproduced from ref. [30] with permission, American Chemical Society) and NWs (d) (reproduced from ref. [31] with permission, Royal Society of Chemistry) 


\subsection{NCG device by adopting unusual structure and Bioinspired approach}

Since the key issues of energy conversion efficiency in composite-based energy harvesters depend on how to uniformly disperse the piezoelectric nanomaterials inside the matrix, it is essential to add the supplementation as filler or dispersant for enhancing the distribution. Recently, Shin et al. described high-performance piezoelectric NCG composed of hemispherically aggregated $\mathrm{BaTiO}_{3} \mathrm{NPs}_{\mathrm{s}}$ and poly-(vinylidene fluoride-co-hexafluoropropene) $[\mathrm{P}(\mathrm{VDF}-\mathrm{HFP})]$ which formed the clusters by solvent evaporation (Fig. 5a) [32-34].The aggregated cluster formation improved the piezoelectric effect by the increment of total dipole moments inside the composite. The hemispherically aggregated $\mathrm{BaTiO}_{3} \mathrm{NPs}$ composite-based NCG device demonstrated the energy generation of $\sim 75 \mathrm{~V}$ and $\sim 15 \mu \mathrm{A}$ at applied pressure of $\sim 0.23 \mathrm{MPa}$. Stretchable composite film-based piezoelectric energy harvester was fabricated by the piezoelectric hemispheres (Fig. 5b) [33]. The highly-ordered piezoelectric hollow hemisphere embedded composite was obtained by the deposition of $\mathrm{ZnO}$ or PZT thin films on a close-packed monolayer polystyrene (PS) beads template. The NCG device consisting of the $10 \mu \mathrm{m}$ hemispheres embedded composite film produced the output voltage of $\sim 4 \mathrm{~V}$ from convex bending which is approximately 8 times higher than outputs under concave motion at same strain $(0.425 \%)$. These results induced by the strong electric dipole alignment inside composite provided the feasibility of the directional anisotropic energy generation with outstanding mechanical stability. Moreover, Jeong et al. [34] demonstrated the NCG device using the unique network of anisotropic and crystalline $\mathrm{BaTiO}_{3}$ nanostructures which are synthesized through the biological self-assembly of multiple metal ions on genetically modified M13 viruses. This fabrication process was mostly performed in an aqueous environment under ambient conditions without toxic chemistry, suggesting an ecofriendly, energy-efficient pathway for the fabrication of a $\mathrm{BaTiO}_{3}$-based NCG. The bio-templated energy harvester produced the electrical outputs up to $\sim 300 \mathrm{nA}$ and $\sim 6 \mathrm{~V}$ which can fully operated the LED-optical fibers and LCD devices without using any additional structural stabilizers and external sources.

\subsection{Hyper-stretchable composite energy harvester}

The stretchability of energy conversion devices also lies in a crucial need to achieve the direct and conformal integration of the stretchable electronic energy sources for various new applications such as electronic skins (e-skins), biomedical devices, and biological sensor network. Although the elastomeric composite-type nanogenerators have been considered as stretchy and freely-deformable piezoelectric energy harvesting systems, the truly reversible and stretchable NCG (strain over $20 \%$ ) has not been realized yet due to the absence of properly-conformal stretchable electrodes with large coverage and the limited elongation of general polymeric matrix. Jeong and coworkers firstly demonstrated an ultra-stretchable piezoelectric energy harvester via the the very long Ag NWs percolation network (VAgNPN) electrodes and ecoflex piezoelectric nanocomposite materials (Fig. 6a) [28]. The very long Ag NWs (VAgNWs) with average length of $\sim 150 \mu \mathrm{m}$ and maximum length of $\sim 500 \mu \mathrm{m}$ were synthesized by a novel successive multistep growth (SMG) method [35]. Using the highly-percolated VAgNWs electrodes after suction transferring onto elastomers, the stretchable electrode exhibited noteworthy conductivity $(\sim 9 \Omega /$ sq) and remarkable stretching strain $(\sim 460 \%)$ without electrical and mechanical failure. Ecoflex silicone rubber was chosen for the matrix of piezoelectric composite because it is hyper-stretchable elastomer up to $\sim 900 \%$. The mixture of $(1-\mathrm{x}) \mathrm{Pb}\left(\mathrm{Mg}_{1 / 3} \mathrm{Nb}_{2 / 3}\right) \mathrm{O}_{3}-\mathrm{x}$ $\mathrm{PbTiO}_{3}$ (PMN-PT) microparticles and MW-CNTs was well blended and dispersed in the silicone rubber. After curing the hyper-stretchable elastomeric p-NC, the VAgNWs were transferred on the $\mathrm{p}-\mathrm{NC}$ by the solution filtration method. By $200 \%$ stretching stimulations, the ultra-stretchable composite generator generated voltage of $\sim 4 \mathrm{~V}$ and current of $\sim 500 \mathrm{nA}$, respectively, which were five times higher than the poor output of the previously-reported mediocre semi-stretchable piezoelectric nanogenerators. Surprisingly, the ultrastretchable piezoelectric generator showed the good mechanical and electrical resistance under diverse mechanical deformations such as twisting, folding (extremely bending), and crumpling (Fig. 6b). Moreover, it could directly produce the electricity signals by all kinds of mechanical stresses.

\subsection{Energy applications by flexible and stretchable NCG devices}

The NCG devices can be used as not only promising energy harvesters that can generate electricity from slight movements by natural and human, but also sensitive sensors which can monitor the weak forces or motions at the wide range of physical and biological fields. Furthermore, the output voltage and currents produced from NCG devices are sufficient to operate the commercial electronic devices and stimulate the animal's nerve. To investigate the potential utilizations of NCG technology, Park et al. [23] realized the energy harvesting that converts human muscle movement such as foot stepping into electrical energy (Fig. 7a). A $\mathrm{BaTiO}_{3} \mathrm{NCG}_{\text {pad driven by }}$ regular and slight pressure results in the repeat energy 

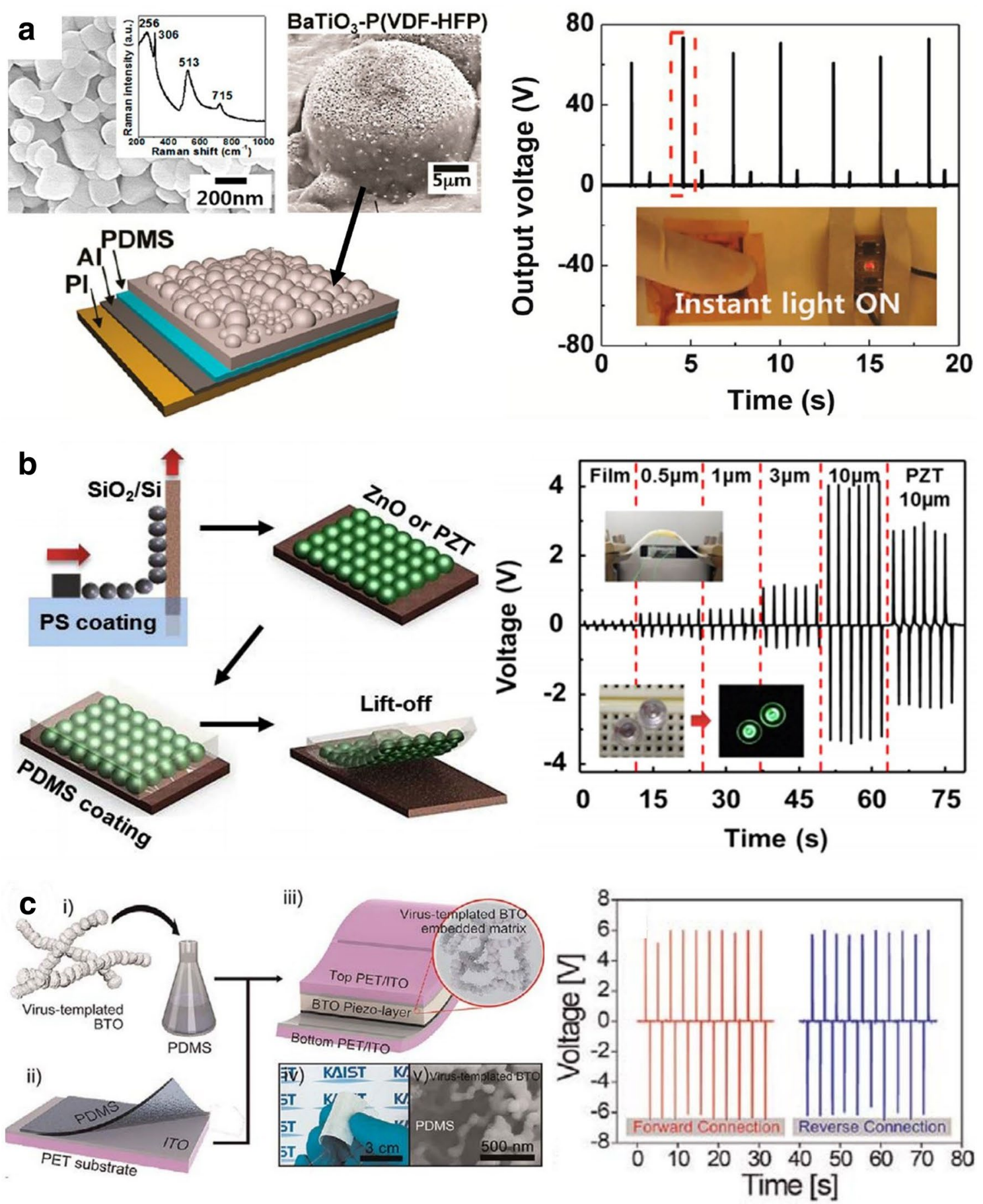

Fig. 5 The NCG devices fabricated by new approaches. a The output voltage harvested from the energy harvester composed of hemispherically aggregated $\mathrm{BaTiO}_{3} \mathrm{NPs} / \mathrm{P}(\mathrm{VDF}-\mathrm{HFP}$ ) polymer(reproduced from ref. [32] with permission, American Chemical Society). b The highly-stretchable composite film-based nanogenerator and its output performance (reproduced from ref. [33] with permission, Elsevier). cThe fabrication process and output voltage of virus-templated $\mathrm{BaTiO}_{3}$ NWs-based NCG device (reproduced from ref. [34] with permission, American Chemical Society)

generation of $\sim 1.5 \mathrm{~V}$ and $\sim 150 \mathrm{nA}$. As shown in Fig. 7b, the commercial liquid crystal display (LCD) screen and light-emitting diodes (LEDs) were simultaneously operated by the solely electricity induced from a PZT particles-based NCG device without any external source [26]. These works showed great potential for NCGs to be 

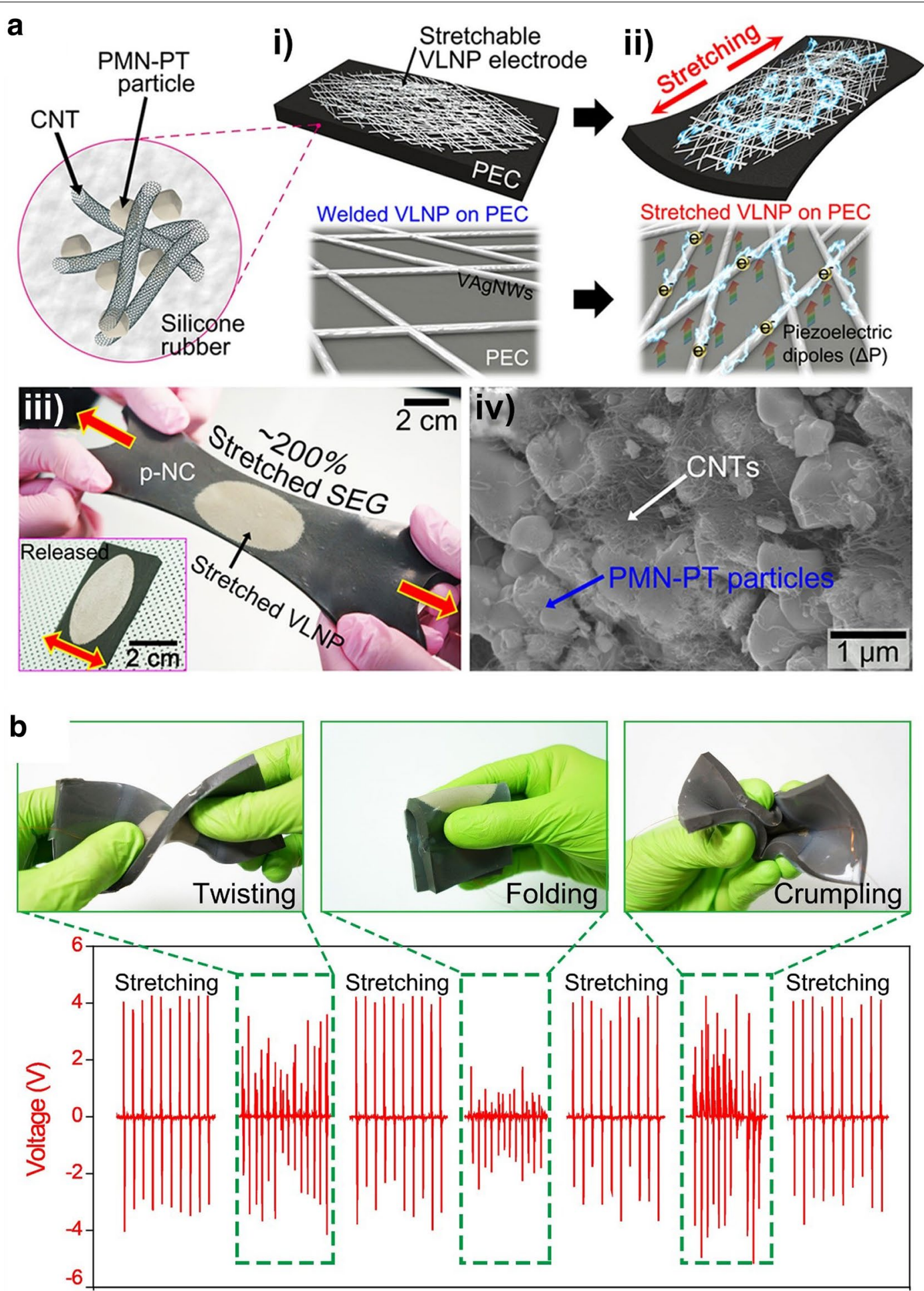

Fig. 6 Hyper-stretchable composite energy harvester. a Schematic diagram of the hyper-stretchable NCG device based on PMN-PT piezoelectric composite and VAgNPN. b The harvested output voltage and current pulse when subjected to various deformations (twisting, folding, and crumpling) (reproduced from ref. [28] with permission, Wiley-VCH)

commercialized in various practical electronic devices. The stimulation of NCG device to nerve is an interesting application of energy harvesting technology. Gu et al. [36] demonstrated the stimulation of frog's sciatic nerve by ultrahigh output power (voltage of $209 \mathrm{~V}$ and current of $17.8 \mu \mathrm{A}$ ) from a PZT NW array-based NCG 

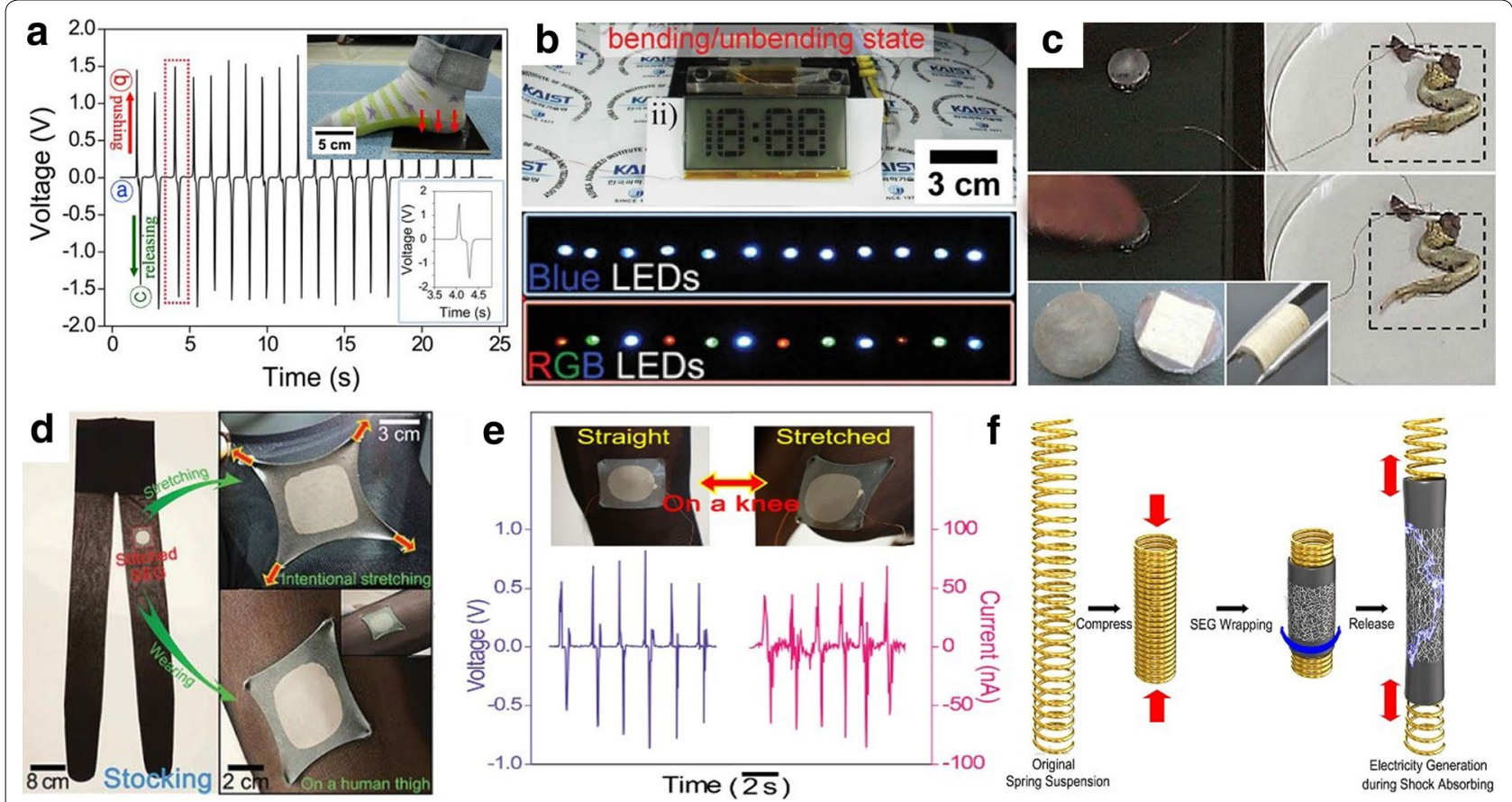

Fig. 7 Energy applications by flexible and stretchable NCG devices. a The electrical energy generated from an NCG pad driven by human muscle movement (foot stepping) (reproduced from ref. [23] with permission, Wiley-VCH). b The commercial electronic devices such as LCD and LEDs operated by the harvested electricity (reproduced from ref. [26] with permission, Wiley-VCH). cThe captured images showing the stimulation of a frog's sciatic nerve by an NCG device (reproduced from ref. [36] with permission, American Chemical Society). d Photographs of the hyper-stretchable NCG stitched on a nylon stocking (reproduced from ref. [28] with permission, Wiley-VCH). e The converted electrical voltage and current from an NCG device under repeatedly stretching (reproduced from ref. [28] with permission, Wiley-VCH). $\mathbf{f}$ The schematic diagram of hyper-stretchable NCG attached on spring-based suspensions. The NCG device can produce power during shock absorbing of spring

device (Fig. 7c). Moreover, the stretchable NCG device was stitched on the stretchy fabrics like a stocking for the wearable energy harvesting system, as shown in Fig. 7d. The inset pictures of Fig. 7e presents the stretched energy harvester by biomechanical kneeling and releasing motions, resulting in periodical voltage signals of $\sim 0.7 \mathrm{~V}$ and current pulses of $\sim 50 \mathrm{nA}$ (Fig. 7e) [28]. This excellent electromechanical compliance of the ultra-stretchable composite nanogenerator would be imagined as energy harvesting modules in transport systems such as automobile spring-based suspensions of seats and frames, as shown in Fig. $7 f$.

\section{Conclusions}

The developments of flexible and stretchable energy harvesting device through piezoelectric materials-polymer composites have enabled the self-powered energy system with high performance and improved mechanical stability. Simple and low-cost fabrication processes such as spin-casing, die-casting, and bar-coating allow the large-area energy harvester. First, the typical piezoelectric nanomaterial (e.g., $\mathrm{BaTiO}_{3} \mathrm{NPs}$ ) was utilized to form the piezoelectric composites; subsequently, the inherently excellent piezoelectric PZT material-based high-output NCG device were developed. Recently, the many researchers have investigated the new approaches for employing the alkaline niobate-based high piezoelectric ceramics and unusual structured $\mathrm{BaTiO}_{3}$ due to additional motivations for environment-friendly and biocompatible energy harvesters. Moreover, the ultrastretchable NCG device were demonstrated via hyperstretchable electrodes of Ag NWs percolation network in response to the demand of energy harvesting devices closely in contact with the curvy surfaces. The fabricated NCG device can produce the power from biomechanical movements and sufficiently operate the commercial electronic devices. The NCG technology will be expected to improve further by adopting the controllable leadfree piezoelectric ceramic materials [such as $\mathrm{Ba}(\mathrm{Zr}, \mathrm{Ti})$ $\mathrm{O}_{3}$ (BZT), (Bi, Nd) $\mathrm{Ti}_{3} \mathrm{O}_{12}$, and $\left.\mathrm{SrBi}_{2} \mathrm{Ta}_{2} \mathrm{O}_{9}\right]$ and the enhaced core-shell structured nanomaterials. Furthermore, the novel printing technique with $\mathrm{p}-\mathrm{NC}$ will open a key role in the development of the commercially available self-powered energy system. The piezoelectric rubbers will facilitate solutions to develop the self-powered road-system consisting of energy generation source, 
highly-sensitive sensor, and wireless transmitter for military and wearable applications. In addition, these unique approaches for flexible energy harvesters shall support energy sources of various state-of-a-art flexible and future electronic systems [37-56].

\section{Authors' contributions}

KIP and CKJ wrote the manuscript and KJL guided manuscript preparation. All authors read and approved the final manuscript.

\section{Authors' information}

Prof. Kwi-ll Park is currently assistant professor in the Department of Energy Engineering at Gyeongnam National University of Science and Technology (GNTECH). Prof. Park received his Ph.D. in Materials Science and Engineering (MSE) at Korea Advanced Institute of Science and Technology (KAIST). During his Ph. D. at KAIST, he studied flexible energy harvesting technology using inorganic-based piezoelectric materials by. He was previously a senior researcher at Agency for Defense Development from 2014 to 2015. His research interests include the synthesis of high performance piezoelectric nanomaterials and development of flexible/stretchable energy harvester based on inorganic piezo-materials.

Dr. Chang Kyu Jeong received his Ph.D. degree in Materials Science and Engineering from KAIST in 2016 and B.S. degree from Hanyang University in 2011. He is currently working as a research fellow in KAIST Institute for the NanoCentury (KINC). His research topics focus on mechanical energy harvesting, soft/biomaterials, surface chemistry, and nanomaterial syntheses.

Prof. Keon Jae Lee received his Ph.D. in Materials Science and Engineering (MSE) at University of Illinois, Urbana-Champaign (UIUC). During his Ph.D. at UIUC, he involved in the first co-invention of "Flexible Single-crystalline Inorganic Electronics", using top-down semiconductors and soft lithographic transfer. Since 2009, he has been a professor in MSE at KAIST. His current research topics are self-powered flexible electronic systems including energy harvesting/storage devices, LEDs, large-scale integration (LSI), high-density memory and laser material interaction for in vivo biomedical and flexible applications.

\section{Author details}

${ }^{1}$ Department of Energy Engineering, Gyeongnam National University of Science and Technology (GNTECH), 33 Dongjin-ro, Jinju-si, Gyeongsangnam-do 52725, Republic of Korea. ${ }^{2}$ Department of Materials Science and Engineering, Korea Advanced Institute of Science and Technology (KAIST), 291 Daehak-ro, Yuseong-gu, Daejeon 34141, Republic of Korea. ${ }^{3}$ KAIST Institute for the NanoCentury (KINC), 291 Daehak-ro, Yuseong-gu, Daejeon 34141, Republic of Korea.

\section{Acknowledgements}

This work was supported by Gyeongnam National University of Science and Technology (GNTECH) Grant 2015. This study was also supported by Global Frontier R\&D Program on Center for Integrated Smart Sensors (Grant No. CISS-2012M3A6A6054193)

\section{Competing interests}

The authors declare that they have no competing interests.

Received: 17 March 2016 Accepted: 12 April 2016

Published online: 03 June 2016

\section{References}

1. G.J. Aubrecht, Energy: physical, environmental, and social impact (Pearson Education, London, 2006)

2. I.R. Henderson, Piezoelectric ceramics: principles and applications (APC International Ltd, Pennsylvania, 2002)

3. S. Priya, D.J. Inman, Energy Harvesting Technologies (Springer Science, New York, 2009)

4. Z.L. Wang, Nanogenerators for self-powered devices and systems (Georgia Institute of Technology, Atlanta, 2011)
5. C.R. Bowen, H.A. Kim, P.M. Weaver, S. Dunn, Piezoelectric and ferroelectric materials and structures for energy harvesting applications. Energ. Environ. Sci. 7, 25-44 (2014)

6. J. Briscoe, S. Dunn, Piezoelectric nanogenerators - a review of nanostructured piezoelectric energy harvesters. Nano. Energ. 14, 15-29 (2015)

7. X. Wang, Piezoelectricnanogenerators-harvesting ambient mechanical energy at the nanometer scale. Nano. Energ. 1(1), 13-24 (2012)

8. Z.L. Wang, W. Wu, Nanotechnology-enabled energy harvesting for selfpowered micro-/nanosystems. Angew. Chem. Int. Ed. 51(47), 1170011721 (2012)

9. S.P. Beeby, M.J. Tudor, N.M. White, Energy harvesting vibration sources for microsystems applications. Meas. Sci. Technol. 17(12), R175 (2006)

10. Z.Y. Wang, J. Hu, A.P. Suryavanshi, K. Yum, M.F. Yu, Voltage generation from individual $\mathrm{BaTiO}_{3}$ nanowires under periodic tensile mechanical load. Nano. Lett. 7(10), 2966-2969 (2007)

11. X.D. Wang, J.H. Song, J. Liu, Z.L. Wang, Direct-current nanogenerator driven by ultrasonic waves. Science. 316(5821), 102-105 (2007)

12. R. Yang, Y. Qin, C. Li, G. Zhu, Z.L. Wang, Converting biomechanical energy into electricity by a muscle-movement-driven nanogenerator. Nano. Lett. 9(3), 1201-1205 (2009)

13. R.S. Yang, Y. Qin, L.M. Dai, Z.L. Wang, Power generation with laterally packaged piezoelectric fine wires. Nat. Nanotechnol. 4(1), 34-39 (2009)

14. G. Zhu, R. Yang, S. Wang, Z.L. Wang, Flexible high-output nanogenerator based on lateral ZnO nanowire array. Nano. Lett. 10(8), 3151-3155 (2010)

15. M.Y. Choi, D. Choi, M.J. Jin, I. Kim, S.H. Kim, J.Y. Choi, S.Y. Lee, J.M. Kim, S.W. Kim, Mechanically powered transparent flexible charge-generating nanodevices with piezoelectric ZnO nanorods. Adv. Mater. 21(21), 2185-2189 (2009)

16. C.E. Chang, V.H. Tran, J.B. Wang, Y.K. Fuh, L.W. Lin, Direct-write piezoelectric polymeric nanogenerator with high energy conversion efficiency. Nano. Lett. 10(2), 726-731 (2010)

17. C.E. Chang, Y-K Fuh, L. Lin, in A direct-write piezoelectric PVDF nanogenerator, transducers 2009, solid-state sensors, actuators and microsystems conference, (Denver, 2009) p. 1485-1488

18. S.N. Cha, S.M. Kim, H. Kim, J. Ku, J.I. Sohn, Y.J. Park, B.G. Song, M.H. Jung, E.K. Lee, B.L. Choi, J.J. Park, Z.L. Wang, J.M. Kim, K. Kim, Porous PVDF as effective sonic wave driven nanogenerators. Nano. Lett. 11(12), 5142-5147 (2011)

19. K.-I. Park, S. Xu, Y. Liu, G.T. Hwang, S.J.L. Kang, Z.L. Wang, K.J. Lee, Piezoelectric $\mathrm{BaTiO}_{3}$ thin film nanogenerator on plastic substrates. Nano. Lett. 10(12), 4939-4943 (2010)

20. Y. Qi, N.T. Jafferis, K. Lyons, C.M. Lee, H. Ahmad, M.C. McAlpine, Piezoelectric ribbons printed onto rubber for flexible energy conversion. Nano. Lett. 10(2), 524-528 (2010)

21. Y. Qi, J. Kim, T.D. Nguyen, B. Lisko, P.K. Purohit, M.C. McAlpine, Enhanced piezoelectricity and stretchability in energy harvesting devices fabricated from buckled PZT ribbons. Nano. Lett. 11(3), 1331-1336 (2011)

22. Y. Qi, M.C. McAlpine, Nanotechnology-enabled flexible and biocompatible energy harvesting. Energ. Environ. Sci. 3(9), 1275-1285 (2010)

23. K.-I. Park, M. Lee, Y. Liu, S. Moon, G.T. Hwang, G. Zhu, J.E. Kim, S.O. Kim, D.K. Kim, Z.L. Wang, K.J. Lee, Flexible nanocomposite generator made of $\mathrm{BaTiO}_{3}$ nanoparticles and graphitic carbons. Adv. Mater. 24(22), 2999-3004 (2012). (Front Cover Article)

24. J.H. Jung, M. Lee, J.I. Hong, Y. Ding, C.Y. Chen, L.J. Chou, Z.L. Wang, Leadfree $\mathrm{NaNbO}_{3}$ nanowires for a high output piezoelectric nanogenerator. ACS. Nano. 5(12), 10041-10046 (2011)

25. J.H. Jung, C.Y. Chen, B.K. Yun, N. Lee, Y. Zhou, W. Jo, L.J. Chou, Z.L. Wang, Lead-free $\mathrm{KNbO}_{3}$ ferroelectric nanorod based flexible nanogenerators and capacitors. Nanotechnology. 23(37), 375401 (2012)

26. K.-I. Park, C.K. Jeong, J. Ryu, G.-T. Hwang, K.J. Lee, Flexible and large-area nanocomposite generators based on lead zirconate titanate particles and carbon nanotubes. Adv. Energ. Mater. 3(12), 1539-1544 (2013)

27. C.K. Jeong, K.-I. Park, J. Ryu, G.-T. Hwang, K.J. Lee, Large-area and flexible lead-free nanocomposite generator using alkaline niobate particles and metal nanorod filler. Adv. Funct. Mater. 24(18), 2620-2629 (2014)

28. C.K. Jeong, J. Lee, S. Han, J. Ryu, G.-T. Hwang, D.Y. Park, J.H. Park, S.S. Lee, M. Byun, S.H. Ko, K.J. Lee, A hyper-stretchable elastic-composite energy harvester. Adv. Mater. 27(18), 2866-2875 (2015)

29. H. Sun, H. Tian, Y. Yang, D. Xie, Y.C. Zhang, X. Liu, S. Ma, H.M. Zhao, T.L. Ren, A novel flexible nanogenerator made of $\mathrm{ZnO}$ nanoparticles and multiwall carbon nanotube. Nanoscale. 5(13), 6117-6123 (2013) 
30. Z.H. Lin, Y. Yang, J.M. Wu, Y. Liu, F. Zhang, Z.L. Wang, BaTiO 3 nanotubesbased flexible and transparent nanogenerators. J. Phys. Chem. Lett. 3(23), 3599-3604 (2012)

31. K.-I. Park, S.B. Bae, S.H. Yang, H.I. Lee, K. Lee, S.J. Lee, Lead-free $\mathrm{BaTiO}_{3}$ nanowires-based flexible nanocomposite generator. Nanoscale. 6(15), 8962-8968 (2014)

32. S.H. Shin, Y.H. Kim, M.H. Lee, J.Y. Jung, J. Nah, Hemispherically aggregated $\mathrm{BaTiO}_{3}$ nanoparticle composite thin film for high-performance flexible piezoelectric nanogenerator. ACS. Nano. 8(3), 2766-2773 (2014)

33. J. Chun, N.-R. Kang, J.-Y. Kim, M.-S. Noh, C.-Y. Kang, D. Choi, S.W. Kaim, Z.L. Wang, J.M. Baik, Highly anisotropic power generation in piezoelectric hemispheres composed stretchable composite film for self-powered motion sensor. Nano. Energ. 11(1), 1-10 (2015)

34. C.K. Jeong, I. Kim, K.-I. Park, M.H. Oh, H. Paik, G.T. Hwang, K. No, Y.S. Nam, K.J. Lee, Virus-directed design of a flexible $\mathrm{BaTiO}_{3}$ nanogenerator. ACS. Nano. 7(12), 11016-11025 (2013)

35. P. Lee, J. Lee, H. Lee, J. Yeo, S. Hong, K.H. Nam, D. Lee, S.S. Lee, S.H. Ko, Highly stretchable and highly conductive metal electrode by very long metal nanowire percolation network. Adv. Mater. 24(25), 3326-3332 (2012)

36. L. Gu, N. Cui, L. Cheng, Q. Xu, S. Bai, M. Yuan, W. Wu, J. Liu, Y. Zhao, F. Ma, Y. Qin, Z.L. Wang, Flexible fiber nanogenerator with $209 \mathrm{~V}$ output voltage directly powers a light-emitting diode. Nano. Lett. 13(1), 91-94 (2013)

37. S.Y. Lee, K.-I. Park, C. Huh, M. Koo, H.G. Yoo, S. Kim, C.S. Ah, G.Y. Sung, K.J. Lee, Water-resistant flexible GaN LED on a liquid crystal polymer substrate for implantable biomedical applications. Nano. Energ. 1, 145-151 (2012)

38. S. Kim, H.Y. Jeong, S.K. Kim, S.-Y. Choi, K.J. Lee, Flexible memristive memory array on plastic substrates. Nano. Lett. 11(12), 5438-5442 (2011)

39. M. Koo, K.-I. Park, S.H. Lee, M. Suh, D.Y. Jeon, J.W. Choi, K. Kang, K.J. Lee, Bendable inorganic thin-film battery for fully flexible electronic systems. Nano. Lett. 12(9), 4810-4816 (2012)

40. W.I. Park, B.K. You, B.H. Mun, H.K. Seo, J.Y. Lee, S. Hosaka, Y. Yin, C.A. Ross, K.J. Lee, Y.S. Jung, Self-assembled incorporation of modulated block copolymer nanostructures in phase-change memory for switching power reduction. ACS. Nano. 7(3), 2651-2658 (2013)

41. G.-T. Hwang, D. Im, S.E. Lee, J. Lee, M. Koo, S.Y. Park, S. Kim, K. Yang, S.J. Kim, K. Lee, K.J. Lee, In vivo silicon-based flexible radio frequency integrated circuits monolithically encapsulated with biocompatible liquid crystal polymers. ACS. Nano. 7(5), 4545-4553 (2013)

42. K.-I. Park, J.H. Son, G.-T. Hwang, C.K. Jeong, J. Ryu, M. Koo, I. Choi, S.H. Lee, M. Byun, Z.L. Wang, K.J. Lee, Highly-efficient, flexible piezoelectric PZT thin film nanogenerator on plastic substrates. Adv. Mater. 26(16), 2514-2520 (2014)

43. G.-T. Hwang, H. Park, J.-H. Lee, K.-I. Park, M. Byun, H. Park, G. Ahn, C.K. Jeong, K. No, H. Kwon, S.-G. Lee, B. Joung, K.J. Lee, Self-powered cardiac pacemaker enabled by flexible single crystalline PMN-PT piezoelectric energy harvester. Adv. Mater. 26(28), 4880-4887 (2014)
44. I. Choi, H.Y. Jeong, D.Y. Jung, M. Byun, C.-G. Choi, B.H. Hong, S.-Y. Choi, K.J. Lee, Laser-induced solid-phase doped graphene. ACS. Nano. 8(8), $7671-7677(2014)$

45. C.K. Jeong, K.-I. Park, J.H. Son, G.-T. Hwang, S.H. Lee, D.Y. Park, H.E. Lee, H.K. Lee, M. Byun, K.J. Lee, Self-powered fully-flexible light-emitting system enabled by flexible energy harvester. Energ. Environ. Sci. 7(12), 4035-4043 (2014)

46. H.S. Lee, J. Chung, G.-T. Hwang, C.K. Jeong, Y. Jung, J.-H. Kwak, H. Kang, M. Byun, W.D. Kim, S. Hur, S.-H. Oh, K.J. Lee, Flexible inorganic piezoelectric acoustic nanosensors for biomimetic artifi cial hair cells. Adv. Funct. Mater. 24(44), 6914-6921 (2014)

47. S. Kim, J.H. Son, S.H. Lee, B.K. You, K.-I. Park, H.K. Lee, M. Byun, K.J. Lee, Flexible crossbar-structured resistive memory arrays on plastic substrates via inorganic-based laser Lift-Off. Adv. Mater. 26(44), 7480-7487 (2014)

48. B.K. You, W.I. Park, J.M. Kim, K.-I. Park, H.K. Seo, J.Y. Lee, Y.S. Jung, K.J. Lee, Reliable control of filament formation in resistive memories by selfassembled nanoinsulators derived from a block copolymer. ACS. Nano. 8(9), 9492-9502 (2014)

49. C.K. Jeong, K.M. Baek, S. Niu, T.W. Nam, Y.H. Hur, D.Y. Park, G.-T. Hwang, M. Byun, Z.L. Wang, Y.S. Jung, K.J. Lee, Topographically-designed triboelectric nanogenerator via block copolymer self-assembly. Nano. Lett. 14(12), 7031-7038 (2014)

50. S.H. Lee, C.K. Jeong, G.-T. Hwang, K.J. Lee, Self-powered flexible inorganic electronic system. Nano. Energ. 14,111-125 (2015)

51. G.-T. Hwang, J. Yang, S.H. Yang, H.-Y. Lee, M. Lee, D.Y. Park, J.H. Han, S.J. Lee, C.K. Jeong, J. Kim, K.-I. Park, K.J. Lee, A reconfigurable rectifi ed flexible energy harvester via solid-state single crystal grown PMN-PZT. Adv. Energ. Mater. 5(10), 1500051 (2015)

52. B.H. Mun, B.K. You, S.R. Yang, H.G. Yoo, J.M. Kim, W.I. Park, Y. Yin, M. Byun, Y.S. Jung, K.J. Lee, Flexible one diode-one phase change memory array enabled by block copolymer self-assembly. ACS. Nano. 9(4), 4120-4128 (2015)

53. H.G. Yoo, M. Byun, C.K. Jeong, K.J. Lee, Performance enhancement of electronic and energy devices via block copolymer self-assembly. Adv. Mater. 27(27), 3982-3998 (2015)

54. B.K. You, M. Byun, S. Kim, K.J. Lee, Self-structured conductive filament nanoheater for chalcogenide phase transition. ACS. Nano. 9(6), 6587-6594 (2015)

55. G.-T. Hwang, Y. Kim, J.-H. Lee, S. Oh, C.K. Jeong, D.Y. Park, J. Ryu, H. Kwon, S.-G. Lee, B. Joung, D. Kim, K.J. Lee, Self-powered deep brain stimulation via a flexible PIMNT energy harvester. Energ. Environ. Sci. 8(9), 2677-2684 (2015)

56. A.H. Park, S.H. Lee, C. Lee, J. Kim, H.E. Lee, S.-B. Paik, K.J. Lee, D. Kim, Optogenetic mapping of functional connectivity in freely moving mice via insertable wrapping electrode array beneath the skull. ACS. Nano. 10(2), 2791-2802 (2016)

\section{Submit your manuscript to a SpringerOpen ${ }^{\odot}$ journal and benefit from:}

- Convenient online submission

- Rigorous peer review

- Immediate publication on acceptance

- Open access: articles freely available online

- High visibility within the field

- Retaining the copyright to your article

Submit your next manuscript at springeropen.com 